\title{
Síndrome de Bartter: una tubulopatía infrecuente de inicio antenatal
}

\author{
Bartter syndrome: an infrequent tubulopathy of prenatal onset
}

\author{
Carmen Luz Gómez de la F. ${ }^{\mathrm{a}}$, José M. Novoa P. a,b, Nury Caviedes R. ${ }^{\mathrm{c}}$
}

aUnidad de Neonatología, Hospital Padre Hurtado. Servicio de Salud Metropolitano Sur-Oriente

bFacultad de Medicina Clínica Alemana de Santiago, Universidad del Desarrollo

'Unidad de Gestión Clínica del Niño, Hospital Padre Hurtado. Servicio de Salud Metropolitano Sur-Oriente

Recibido: 29 de noviembre de 2018; Aceptado: 11 de marzo de 2019

\begin{abstract}
Resumen
Introducción: Síndrome de Bartter (SB) es una tubulopatía hereditaria, poco frecuente que tiene dos formas de presentación, forma grave de inicio antenatal (Bartter neonatal) y forma de aparición más tardía (Bartter clásico). En su forma antenatal se manifiesta con poliuria fetal, polihidroamnios de inicio precoz y severo, parto prematuro secundario y restricción de crecimiento intrauterino. La etapa postnatal presenta episodios recurrentes de deshidratación y desbalance electrolítico que pueden comprometer la sobrevida del paciente. Objetivo: Comunicar un caso de SB neonatal y presentar una revisión de la literatura en esta patología. Caso Clínico: Prematuro 35 semanas, con antecedente de severo polihidroamnios diagnosticado a las 27 semanas de gestación, sin causa aparente. Desde su nacimiento evolucionó con poliuria y alcalosis metabólica hipokalémica haciendo plantear, en primera semana de vida, diagnóstico de Síndrome de Bartter neonatal. El laboratorio confirmó perdidas urinarias de electrólitos. Fue manejado con balance hídrico estricto y suplementación de sodio y potasio, logrando estabilizar peso y desbalance electrolítico. Se mantiene en control nefrológico, con suplementación de gluconato de potasio y cloruro de sodio. Se agregó ibuprofeno al cuarto mes como parte del tratamiento. $\mathrm{Al}$ séptimo mes de vida, ecografía renal demostró nefrocalcinosis. $\mathrm{Al}$ año de vida se evidenció hipoacusia sensorioneural profunda requiriendo implante coclear. Conclusión: Presencia de polihidroamnios severo de aparición temprana sin causa identificada debe hacer sospechar SB, que aun siendo infrecuente determina graves alteraciones hidroelectrolíticas y debe ser iniciado su tratamiento precozmente.
\end{abstract}

\begin{abstract}
Introduction: Bartter syndrome (BS) is a rare inherited tubulopathy that has two presentation forms, the first one is a severe form of antenatal onset (neonatal Bartter) and the second one is a later onset form during the first years of life (classic Bartter). In the antenatal form, it manifests with fetal polyuria, polyhydramnios of early and severe onset, premature delivery, and intrauterine growth
\end{abstract}

Palabras clave: Síndrome de Bartter; polihidroamnios; alcalosis metabólica hipokalémica; tubulopatía

\section{Keywords:}

Bartter syndrome; polyhydramnios; hypokalemic metabolic alkalosis; tubulopathy

Correspondencia:

Carmen Luz Gómez de la Fuente

cluzgomez@gmail.com

Como citar este artículo: Rev Chil Pediatr 2019;90(4):437-442. DOI: 10.32641/rchped.v90i4.932 
restriction. In the postnatal stage, it presents recurrent episodes of dehydration and electrolyte imbalance that can compromise the survival of the patient. Objective: To report a clinical case of neonatal BS and a review of the literature. Clinical Case: Premature newborn of 35 weeks of gestation with history of severe polyhydramnios diagnosed at 27 weeks of gestation, without apparent cause. From birth, the patient presented polyuria and hypokalemic metabolic alkalosis making a diagnosis of Neonatal Bartter Syndrome in the first week of life. Laboratory tests confirmed urinary electrolyte losses. The patient was treated with strict water balance and sodium and potassium supplementation, achieving weight and electrolyte imbalance stabilization. The patient remains in control in the nephrology unit, with potassium gluconate and sodium chloride supplementation. At the fourth month, ibuprofen was added as part of treatment. At the seventh month of life, renal ultrasound showed nephrocalcinosis. At one year of life, profound sensorineural hearing loss was observed requiring a cochlear implant. Conclusion: The presence of severe polyhydramnios of early onset with no identified cause should lead to suspicion of neonatal BS which even when infrequent determines severe hydroelectrolytic alterations and should be treated early.

\section{Introducción}

El Síndrome de Bartter (SB) es una tubulopatía hereditaria, poco frecuente que tiene dos formas de presentación, una forma grave de inicio antenatal (Bartter neonatal) y una forma de aparición más tardía, durante los primeros años de la vida (Bartter clásico). Este síndrome es parte de un grupo genéticamente heterogéneo y poco frecuente de entidades definidas por anomalías de la función tubular renal que se heredan con un patrón autosómico recesivo. El mecanismo patogénico primario es el defecto en reabsorción de sodio y cloro en la porción gruesa del asa ascendente de Henle.

Fue descrito en la década del 60 por Bartter y col. ${ }^{1}$ quienes reportaron una nefropatía perdedora de sal caracterizada por hipokalemia, alcalosis metabólica, poliuria e hipertrofia del aparato yuxtaglomerular. Avances en genética y biología molecular permitieron conocer que se trata de un trastorno genético heterogéneo provocado por defecto en reabsorción de sodio, cloro y potasio en la porción gruesa de la rama ascendente de Henle.

Se caracteriza por poliuria asociada a hipokalemia, hipocloremia, alcalosis metabólica, hipercalciuria e hiperaldosteronismo hiperreninémico secundario, con presión arterial normal o baja ${ }^{1}$.

Aunque se han descrito 7 variantes genéticas, sólo se distinguen dos formas clínicas de la enfermedad. Una forma de inicio prenatal caracterizada por polihidroamnios y parto prematuro, presentándose el neonato con clínica de deshidratación severa por poliuria en los primeros días de vida, evolucionando precozmente con nefrocalcinosis y alteraciones bioquímicas características (SB neonatal), y una forma menos severa llamada SB clásico que debuta más tardíamente, generalmente en los primeros dos años de vida, con déficit de crecimiento y episodios recidivantes de deshidratación ${ }^{2,3}$. La incidencia exacta de SB es desconoci- da, pero se presume de 1,2/1.000.000 nacidos ${ }^{4}$. El objetivo es informar un caso clínico de paciente con SB neonatal y presentar una revisión de la patología.

\section{Caso clínico}

Neonato femenino, segundo hijo de padres no consanguíneos. Madre 26 años, sana, derivada a maternidad a las 27 semanas por polihidramnios severo con índice de líquido amniótico (ILA) 41,7 siendo ILA > 24 indicador de polihidramnios y $>40$ polihidroamnios severo. Se descartan malformaciones fetales, diabetes materna y patología placentaria. A las 35 semanas de embarazo presentó ILA 54. Por deterioro de unidad fetoplacentaria, nació vía cesárea a las 35 semanas, Apgar 9-9, peso $2.180 \mathrm{~g}$ y talla $47 \mathrm{~cm}$. Sin dismorfías y tono muscular normal. Se descartó atresia esofágica. Evolucionó con dificultad respiratoria con bajos requerimientos de oxígeno.

Cursó precozmente con poliuria significativa $(7,1$ $\mathrm{ml} / \mathrm{kg} / \mathrm{h}$ ). A las $24 \mathrm{hrs}$ de vida natremia $136 \mathrm{mEq} / \mathrm{l}$, kalemia $4,0 \mathrm{mEq} / \mathrm{l}$, hipocloremia $89 \mathrm{mEq} / \mathrm{l}$, alcalosis metabólica pH 7,45; bicarbonato 27,2 mmol/l. Pérdida de peso fue $6 \%$ a las $24 \mathrm{~h}$, acentuándose hasta alcanzar una baja de $19 \%$ al $10^{\circ}$ día. Se manejó con aportes hídrico entre $150-184 \mathrm{ml} / \mathrm{kg} /$ día, sodio hasta $7,6 \mathrm{mEq} /$ $\mathrm{kg} /$ día y potasio de $3,6 \mathrm{mEq} / \mathrm{kg} /$ día.

Evolucionó con tendencia a hiponatremia, alcalosis metabólica hipocloremica e hipokalemica ( $\mathrm{pH} 7,47$; bicarbonato $30 \mathrm{mmol} / \mathrm{l}$; sodio $123 \mathrm{mEq} / \mathrm{l}$; potasio 2,2 $\mathrm{mlEq} / \mathrm{l}$; cloro $75 \mathrm{mEq} / \mathrm{l})$. Calcemia y magnesemia normales. Nitrógeno ureico y creatinemia normales. Presión arterial normal.

$\mathrm{Al} 5^{\circ}$ día presentó pérdidas urinarias elevadas de sodio $(\mathrm{FeNa} 25 \%$ ) y potasio (Fe K $32.4 \%$ ). Relación calciuria / creatinuria de 0,99 (Normal para edad $<0,8$ ).

Considerando evolución clínica y de laboratorio se 
planteó diagnóstico de SB neonatal. Se manejó con balance hídrico estricto, aportes de sodio 6,5 a 7,6 mEq/ $\mathrm{kg} /$ día y de potasio 3 a $3,6 \mathrm{mEq} / \mathrm{kg} /$ día, logrando estabilizar peso y electrolitos, manteniendo diuresis entre 3-5 $\mathrm{ml} / \mathrm{kg} / \mathrm{h}$. Al $18^{\circ}$ día logró aporte oral exclusivo y se mantuvo suplementación enteral de sodio y potasio, bien tolerados. Recuperó peso de nacimiento al $20^{\circ}$ día de vida.

Ecografía renal al quinto día fue normal, repetida los 27 días describió a nivel de pirámides fino halo hiperecogénico sugerente de nefrocalcinosis. Screening auditivo BERA fue normal al $3^{\circ}$ día de vida.

Egresó a los 35 días, con indicación de suplementación oral de $\mathrm{NaCl} 6,4 \mathrm{mEq} / \mathrm{kg} /$ día y gluconato de potasio $3,1 \mathrm{mEq} / \mathrm{kg} /$ día.

En control en Nefrología, con suplementación de gluconato de potasio, debiendo aumentarse de 2 a 3 $\mathrm{mEq} / \mathrm{kg} /$ día a los tres meses por tendencia a hipokalemia. Natremia normal con aportes de cloruro de sodio de $3,8 \mathrm{mEq} / \mathrm{kg} /$ día. Cursó con alcalosis metabólica, bicarbonato de 31,6 mmol/l. Se agregó ibuprofeno al cuarto mes. Evolucionó con lento progreso pondoestatural e hipotonía. Evaluación nutricional al $6^{\circ}$ mes confirmó desnutrición crónica moderada. Ecografía renal al $7^{\circ}$ mes revela nefrocalcinosis. $\mathrm{Al}$ año de vida se evidenció hipoacusia sensorioneural profunda requiriendo implante coclear.

\section{Discusión}

Las mutaciones de varios genes que codifican los transportadores involucrados en la reabsorción de sal en rama gruesa ascendente asa de Henle causan diferentes tipos de SB que se clasifican según las alteraciones genéticas involucradas (tabla 1). El tipo I o SB antenatal, conocido anteriormente como Síndrome de Hiperprostaglandina E causado por mutación del gen SLC12A1 en cromosoma $15 q 15-21^{5,6}$ que codifica el cotransportador de NKCC2 (NaK2Cl), sensible a furosemida, en el túbulo renal y responsable de la reabsorción de alrededor del $30 \%$ del $\mathrm{NaCl}$ filtrado. Estas anormalidades bioquímicas son similares a las inducidas por terapia crónica con furosemida.

El SB tipo II también denominado junto al tipo I, $\mathrm{SB}$ antenatal, presenta mutación del gen KCNJ1 que codifica canal ROMK, el cual recicla y reabsorbe el potasio hacia la luz tubular ${ }^{7}$. Cuando se pierde la capacidad de reciclar potasio desde las células hacia la luz tubular, la concentración luminal de potasio está demasiado reducida para permitir la actividad del cotransportador $\mathrm{Na}-\mathrm{K}-2 \mathrm{Cl}$. Este canal potásico (ROMK) también se expresa en células del túbulo colector y estos pacientes pueden presentar inicialmente una hiperkalemia transitoria y posteriormente evolucionar con hipokalemia.

Tabla 1. Características de los diferentes tipos de síndrome de Bartter

\begin{tabular}{|c|c|c|c|c|c|}
\hline Tipo & $\begin{array}{l}\text { Gen } \\
\text { involucrado }\end{array}$ & $\begin{array}{l}\text { Proteína } \\
\text { cotransportadora } \\
\text { afectada }\end{array}$ & Herencia & Polihidroamnios & Presentación clínica \\
\hline Bartter tipo I & SLC12A1 & NKCC2 & $\begin{array}{l}\text { Autosómico } \\
\text { recesivo }\end{array}$ & Muy frecuente & $\begin{array}{l}\text { Presentación antenatal. Poliuria, Hipoclo- } \\
\text { remia, hipokalemia, alcalosis metabólica, } \\
\text { nefrocalcinosis }\end{array}$ \\
\hline Bartter tipo II & KCNJ1 & ROMK & $\begin{array}{l}\text { Autosómico } \\
\text { recesivo }\end{array}$ & Muy frecuente & $\begin{array}{l}\text { Presentación antenatal. Hiperkalemia inicial } \\
\text { transitoria ( } 66 \% \text { de los casos), hipokalemia } \\
\text { posterior. Poliuria, hipocloremia, alcalosis } \\
\text { metabólica, nefrocalcinosis }\end{array}$ \\
\hline Bartter tipo III & $\mathrm{CLC}-\mathrm{Kb}$ & CLC-Kb & $\begin{array}{l}\text { Autosómico } \\
\text { recesivo }\end{array}$ & Infrecuente & $\begin{array}{l}\text { Presentación 0-5 años (Clásico o antenatal). } \\
\text { Hipocloremia, hipokalemia, alcalosis meta- } \\
\text { bólica. Hipomagnesemia. Gran variabilidad } \\
\text { clínica. Nefrocalcinosis infrecuente }\end{array}$ \\
\hline Bartter tipo IVA & BSND & Barttina & $\begin{array}{l}\text { Autosómico } \\
\text { recesivo }\end{array}$ & Muy frecuente & \multirow{2}{*}{$\begin{array}{l}\text { Presentación antenatal. Poliuria, hipoclo- } \\
\text { remia, hipokalemia, alcalosis metabólica. } \\
\text { Sordera neurosensorial. Nefrocalcinosis } \\
\text { infrecuente }\end{array}$} \\
\hline Bartter tipo IVB & $\begin{array}{l}\text { CLC-Ka y } \\
\text { CLC-Kb }\end{array}$ & $\begin{array}{l}\text { CLC-Ka y } \\
\text { CLC-Kb }\end{array}$ & $\begin{array}{l}\text { Autosómico } \\
\text { recesivo. Digénico }\end{array}$ & & \\
\hline Bartter tipo V & CaSR & CaSR & $\begin{array}{l}\text { Autosómico } \\
\text { dominante }\end{array}$ & No presenta & $\begin{array}{l}\text { Síndrome de Bartter con hipocalcemia, } \\
\text { hipokalemia, hipomagnesemia, nefrocalci- } \\
\text { nosis. Hipoparatiroidismo }\end{array}$ \\
\hline $\begin{array}{l}\text { Bartter } \\
\text { transitorio }\end{array}$ & MAGE-D2 & MAGE-D2 & $\begin{array}{l}\text { Ligada } X, \\
\text { recesiva }\end{array}$ & Presente & $\begin{array}{l}\text { Presentación antenatal. Poliuria, alteraciones } \\
\text { hidro-electrolíticas transitorias }\end{array}$ \\
\hline
\end{tabular}


El SB clásico o tipo III causado por mutación en gen que codifica para canal de cloro CLC-Kb situado en cromosoma 1 p36 es de gran variedad fenotípica, puede originar presentaciones antenatales o simular un síndrome de Gitelman con hipocalciuria e hipomagnesemia ${ }^{8-10}$. Un defecto funcional del canal de cloro (CLC-Kb) se acompaña secundariamente de reducción de reabsorción tubular de $\mathrm{NaCl}$. Debido a que en el lado basolateral existe otro canal de cloro, ClC-Ka, es probable que la pérdida de $\mathrm{NaCl}$ sea menor que en las variantes neonatales y se produzca una menor eliminación urinaria de calcio y menor probabilidad de nefrocalcinosis.

El SB tipo IVA, se presenta con forma antenatal severa acompañado de sordera neurosensorial ${ }^{11}$ debido a la mutación del gen BSND que codifica para la proteína llamada Barttina, esencial para el correcto funcionamiento de canales de cloro CLC-Ka y CLC Kb, que se encuentran en la membrana basolateral de túbulos renales y también en el epitelio secretor de potasio del oído interno, por lo que la mutación de este gen produce también incapacidad para secreción de potasio dentro de la endolinfa lo, que explica el compromiso auditivo.

Se ha reportado un subtipo adicional de SB con sordera neurosensorial que no presenta mutación en el gen que codifica para la Barttina, pero si presenta mutaciones heterocigotas (herencia digénica) en los dos genes que codifican para los canales de cloro CLC-Ka y CLC Kb que se ha denominado SB tipo IVB ${ }^{12}$.

El SB tipo V, único de herencia autosómica dominante, producido por mutaciones de ganancia de función en el gen CASR que codifica para receptor sensible al calcio, en la membrana basolateral de células de porción gruesa ascendente del asa de Henle. No se asocia con SB antenatal ${ }^{13}$. En 2016, Laghmani describió la mutación por perdida de función del gen MAGED2, asociado a una forma grave pero transitoria de SB antenatal ligado al cromosoma X (OMIM 300971) $)^{14}$. En figura 1 se esquematizan los trastornos involucrados en los diferentes tipos de SB.

En condiciones fisiológicas la reabsorción de iones en la porción gruesa ascendente del asa de Henle es un proceso extremadamente complejo que requiere indemnidad de los diferentes canales y co-transportadores en la célula tubular. Defecto en cualquiera de ellos provoca pérdida renal de sodio, cloro, potasio y calcio que va a tratar de compensarse en otros segmentos del túbulo. La manifestación más temprana de esta disfunción tubular es poliuria fetal que conduce en último trimestre del embarazo a desarrollar severo polihidroamnios.

Diagnóstico antenatal es posible mediante documentación de niveles elevados de cloro en líquido amniótico y estudio genético ${ }^{15}$.
La consecuencia directa del defecto molecular en porción gruesa ascendente del asa de Henle (TALH) es una falla en reabsorción de sodio filtrado. La cantidad elevada de sodio que llega a la nefrona distal de los túbulos excede la posibilidad de compensación por el túbulo contorneado distal y los conductos colectores provocando pérdida de sodio. La pérdida crónica de sodio lleva a contracción del volumen extracelular e hipovolemia secundaria, con activación del sistema renina-angiotensina-aldosterona que estimula la reabsorción de sodio en la célula principal de los conductos colectores, lo que se acompaña de excreción de potasio e hidrógeno causando alcalosis metabólica hipopotasémica. El transportador NKCC2, así como el canal de potasio ROMK también se expresan en la macula densa y la deficiencia va a alterar la detección de sales en el filtrado tubular. Esto agrava un estado hiperreninémico ya estimulado por la pérdida de sal y la contracción del volumen.

La prostaglandina E2 se libera en respuesta al deterioro de la absorción de sodio en TALH y se puede encontrar niveles altos en orina y sangre, pero el modo en que agrava la perdida de sal y agua renal no está completamente resuelto. Los inhibidores de la síntesis de prostaglandinas, son eficaces en la supresión de la perdida de sal y agua y dan como resultado que los niños afectados a menudo alcancen tasas de crecimiento normales ${ }^{16-18}$.

La asociación de hipercalciuria se explica porque alrededor del 25\% de calcio filtrado es reabsorbido en la rama ascendente gruesa del asa de Henle acoplado a la actividad de NKCC2. La reabsorción paracelular de calcio es movida por un voltaje transepitelial luz positivo. Existen 2 prerrequisitos para generar este gradiente de voltaje transepitelial: 1) un importante gradiente transepitelial de $\mathrm{ClNa}$ dependiente de la acción coordinada del cotransportador $\mathrm{Na} / \mathrm{K} / 2 \mathrm{Cl}$ (NKCC2), el canal de potasio ROMK, ambos en la membrana apical, y el canal de cloro (ClCKb-barttina) en la membrana basolateral; 2) un canal paracelular catión-selectivo dependiente de la interacción de claudina 16, 19 y $14^{19}$. La abolición del potencial transtubular provocado por el defecto en los cotransportadores se traduce en incapacidad para conducir pasivamente cationes a través del espacio intercelular en asa de Henle (figura 1).

En SB, a pesar de cursar con hiperaldosteronismo la presión arterial es normal por su estado de hipovolemia y niveles elevados de PGE2.

Teniendo en cuenta la fisiopatología del SB, la inhibición de la síntesis de prostaglandinas a nivel renal y sistémico por los antiinflamatorios no esteroidales es fundamental para el control de la enfermedad.

Se ha descrito el uso de indometacina en etapa prenatal para manejo del polihidroamnios antes de las 


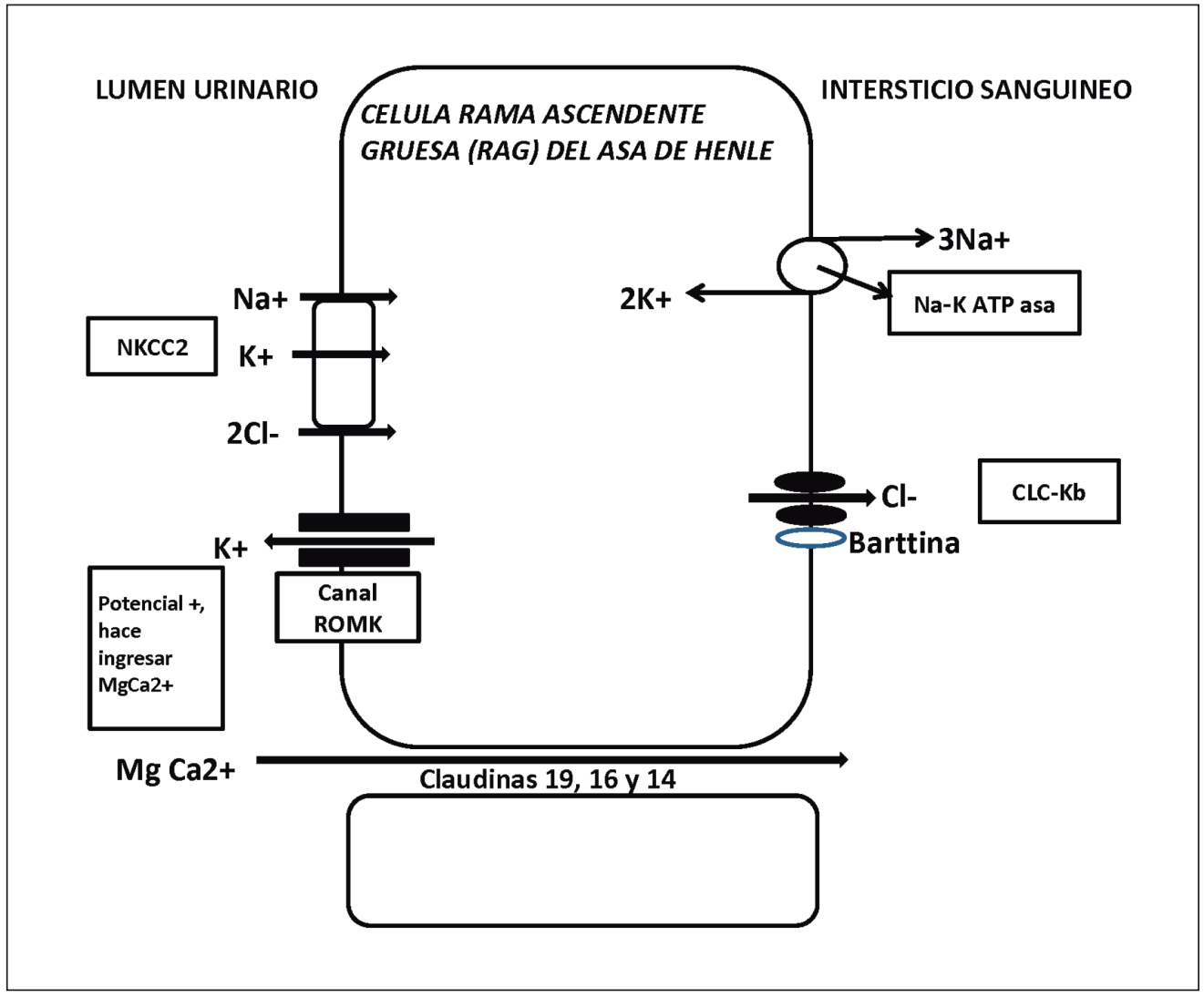

Figura 1. Célula RAG del Asa de Henle. Co-transportadores afectados en el Sindrome de Bartter.

32 semanas de embarazo, pero se debe considerar sus efectos en el cierre ductal con riesgo de hipertensión pulmonar postnatal que obliga a una monitorización estricta $^{20}$.

En la etapa postnatal los inhibidores de síntesis de prostaglandinas contribuyen a disminuir la poliuria, incrementan el potasio sérico, reducen la hipercalciuria, pero no previenen la nefrocalcinosis ${ }^{21}$.

Los inhibidores selectivos de COX-2 han demostrado ser tan efectivos como los no selectivos, en la reducción de poliuria en SB antenatal ${ }^{22}$. Sin embargo, no hay suficiente experiencia en uso de estos fármacos en etapa neonatal. La corrección cuidadosa del desbalance hidroelectrolítico es el aspecto fundamental del manejo en estos pacientes ${ }^{23}$.

En nuestro caso el diagnóstico se basó en antecedente de polihidramnios severo y en la etapa postnatal por la presencia de poliuria y alteraciones hidroelectrolíticas con hipokalemia severa, alcalosis metabólica persistente y nefrocalcinosis. La hipoacusia sensorioneural profunda en este caso orientaría a SB tipo IV. El estudio genético no estuvo disponible en nuestro caso por limitación económica. Teniendo en cuenta la fisiopatología del SB, un elemento esencial del manejo pre y postnatal es la inhibición de síntesis de prostaglandinas a nivel renal y sistémico con antiinflamatorios no esteroidales.

\section{Conclusión}

Presencia de polihidroamnios severo de aparición temprana sin causa habitual como diabetes materna o anomalía gastrointestinal fetal debe hacer sospechar $\mathrm{SB}$, que aún cuando es infrecuente determina graves alteraciones hidroelectrolíticas neonatales.

\section{Responsabilidades Éticas}

Protección de personas y animales: Los autores declaran que los procedimientos seguidos se conformaron a las normas éticas del comité de experimentación humana responsable y de acuerdo con la Asociación Médica Mundial y la Declaración de Helsinki.

Confidencialidad de los datos: Los autores declaran que han seguido los protocolos de su centro de trabajo sobre la publicación de datos de pacientes.

Derecho a la Privacidad y Consentimiento Informado: Los autores declaran que la información ha sido obtenida de datos previos en forma anonimizada, por lo cual el Comité de Ética de Investigación en uso de sus facultades, ha eximido de la obtención de un consentimiento informado, lo cual consta en el acta respectiva.

\section{Conflicto de intereses}

Los autores declaran no tener conflicto de intereses. 


\section{Referencias}

1. Bartter FC, Pronove P, Gill JR Jr, MacCardle RC, Diller E. Hyperplasia of the yuxtaglomerular complex with hyperaldosteronism and hypokalemic alkalosis. Am J Med 1962; 33:811-28.

2. Brochard K, Boyer O, Blanchard A, et al. Phenotype-geno-type correlation in antenatal and neonatal variants of Bartter syndrome. Nephrol Dial Transplant 2009; 24(5):1455-64.

3. Seyberth HW, Schlingmann KP. Bartter and Gitelman-like. syndromes: salt-losing tubulopathies with loop or DCT defects. Pediatr Nephrol 2011; 26:1789-802.

4. Rudin A. Bartter's syndrome. A review of 28 patients followed for 10 years. Acta Med Scand 1988; 224: 165-71.

5. Simon DB, Karet FE, Hamdan JM, Di Pietro A, Sanjad SA, Lifton RP. Bartter's syndrome, hypokalaemic alkalosis with hypercalciuria, is caused by mutations in the $\mathrm{Na}-\mathrm{K}-2 \mathrm{Cl}$ cotransporter NKCC2. Nat Genet. 1996;13:183-8.

6. Starremans PG, Kersten FF, Knoers NV, van den Heuvel LP, Bindels RJ. Mutations in the human $\mathrm{Na}-\mathrm{K}-2 \mathrm{Cl}$ cotransporter (NKCC2) identified in Bartter syndrome type I consistently result in nonfunctional transporters. J Am Soc Nephrol. 2003;14:1419-26.

7. Finer G, Shalev H, Birk OS, Galron D, Jeck N, Sinai-Treiman L. Transient neonatal hyperkalemia in the antenatal (ROMK defective) Bartter syndrome Pediat 2003;142(3):318-23.

8. Rodríguez-Soriano J, Vallo A, Pérez de
Nanclares G, Bilbao JR, Castaño L. A founder mutation in the CLCNKB gene causes Bartter syndrome type III in Spain. PediatrNephrol 2005; 20: 891-6.

9. Konrad M, Vollmer M, Lemmink HH, et al. Mutations in the chloride channel gene CLCNKB as a cause of classic Bartter syndrome. J Am Soc Nephrol 2000;11(8):1449-59.

10. Jeck N, Konrad M, Peters M, Weber S, Bonzel KE, Seyberth HW. Mutations in the chloride channel gene, CLCNKB, leading to a mixed Bartter-Gitelman phenotype. Pediatr Res 2000;48(6): 754-8.

11. Birkenhager R, Otto E, Schurmann MJ, et al. Mutation of BSND causes Bartter syndrome with sensorineural deafness and kidney failure. Nat Genet 2001;29(3):3104.

12. Schlingmann KP, Konrad M, Jeck N, Waldegger P, Reinalter SC, Holder M, Seyberth HW, Waldegger S. Salt wasting and deafness resulting from mutations in two chloride channels. N Engl J Med 2004; 350:1314-9.

13. Watanabe $\mathrm{S}$, Fukumoto $\mathrm{S}$, Chang $\mathrm{H}$, et al. Association between activating mutations of calcium-sensing receptor and Bartter's Syndrome.Lancet 2002;360:692-4.

14. Laghmani K, Beck BB, Yang S-S, et al. Polyhydramnios, transient antenatal Bartter's syndrome, and MAGED2 mutations. N Engl J Med 2016; 374:185363.

15. Dane B, Mayla M, Rane C, Cetin A. Prenatal diagnosis of Bartter syndrome with biochemical examination of amniotic fluid. Fetal Diagn Therap 2007; 22 : 206-8.

16. Stokes JB. Effect of prostaglandin E2 on chloride transport across the rabbit thick ascending limb of Henle. Selective inhibitions of the medullary portion. J Clin Invest 1979; 64:495-502.

17. Konrad M, Leonhardt A, Hensen P, Seyberth WH, Kockerling A. Prenatal and postnatal management of Hyperprostaglandin E syndrome after genetic diagnosis from amniocytes. Pediatrics 1999;103:678-83.

18. RM Nüsing,HW Seiberth.The role of cyclooxygenases and prostanoid receptors in furosemide-like salt losing tubulopathy: the hyperprostaglandin E syndrome. Acta Physiol Scand. 2004;181:523-8.

19. Negri A. Rol de las claudinas en el manejo renal del calcio.Nefrologia (Madr.) 2015;35:347-52. doi: 10.1016/j. nefro.2015.06.011.

20. Drukker A. The adverse renal effects of prostaglandin-synthesis inhibition in the fetus and the newborn. Paediatr Child Health. 2002;7(8):538-43.

21. Vaisbich MH, Fujimura MD, Koch VH. Bartter syndrome: benefits and side effects of long-term treatment. Pediatr Nephrol 2004;19:858-63.

22. Nascimento CL, García CL, Schvartsman B, Vaisbich MH. Treatment of Bartter syndrome. Unsolved issue. J Pediatr (Rio J) 2014;90(5):512-7.

23. Azzi A, Chehade Deschenes G. Neonates with Bartter syndrome have enormous fluid and sodium requirements. Acta Paediatr. 2015;104(7):e294-299. 